\title{
10 Years of Multi-Frequency Monitoring of Blazars with Medicina and Noto
}

\author{
U. Bach ${ }^{* a}$, M. Giroletti ${ }^{b}$, P. Leto ${ }^{c}$, C.M. Raiteri ${ }^{d}$, C. Buemi ${ }^{c}$, F. D’Ammando ${ }^{b, e}$, L.

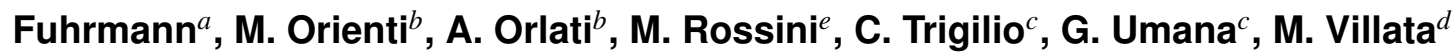 \\ ${ }^{a}$ Max-Planck-Institut fuer Radioastronomie, Bonn, Germany \\ ${ }^{b}$ Istituto di Radioastronomia, INAF, Bologna, Italy \\ ${ }^{c}$ Osservatorio Astrofisico di Catania, INAF, Catania, Italy \\ ${ }^{d}$ Osservatorio Astrofisico di Torino, INAF, Pino Torinese, Itay \\ e Universita di Bologna, Bologna, Italy \\ E-mail: ubach@mpifr.de, girolettieira.inaf.it, \\ paolo.leto@oact.inaf.it, raiteri@oato.inaf.it, \\ carla.buemi@oact.inaf.it, dammando@ira.inaf.it, lfuhrmann@mpifr.de, \\ orienti@ira.inaf.it, a.orlati@ira.inaf.it, \\ matteo.rossini2@studio.unibo.it, corrado.trigilio@oact.inaf.it, \\ grazia.umana@oact.inaf.it, villata@oato.inaf.it
}

\begin{abstract}
Being dominated by non-thermal emission from aligned relativistic jets, blazars allow us to elucidate the physics of extra-galactic jets, and, ultimately, how the energy is extracted from the central black hole in radio-loud AGN. Crucial information is provided by their spectral energy distribution (SED) from radio to gamma-rays, their trends with luminosity and correlated multi-frequency variability. Therefore a monthly monitoring of the broad-band radio spectra at frequencies of 5 , 8, 22, and $43 \mathrm{GHz}$ was proposed in October 2004 at the telescopes in Medicina and Noto by the GLAST-AGILE Support Program (GASP) of the Whole Earth Blazar Telescope (WEBT). We organized contemporaneous near-IR and optical observations for most observing epochs which provides a valuable database of time dependent SEDs of our sample.
\end{abstract}

12th European VLBI Network Symposium and Users Meeting

7-10 October 2014

Cagliari, Italy

${ }^{*}$ Speaker. 


\section{Introduction}

The term Blazars identifies a family of radio-loud Active Galactic Nuclei (AGN) showing a rather complex phenomenology: extreme variability at all wavelengths, polarization, strong $\gamma$-ray emission and brightness temperatures exceeding the Compton limit ([23]). The large amount of work done in the last decade has led to a rather general consensus on the global mechanism responsible for the emission: a rotating black hole surrounded by a massive accretion disk with an intense plasma jet closely aligned to the line of sight. Relativistic electrons produce the soft photons through synchrotron emission, while hard photons are likely produced by inverse Compton scattering. This overall scenario, however, still presents a large number of poorly understood details which, in turn, lead to a wide variety of models and call for long term and multi-wavelength campaigns capable of providing the necessary observational constraints.

Variability measurements provide key information on the AGN structure, down to linear scales or flux density levels not accessible even with interferometric imaging. The mechanisms for variability are still not well understood. Possibilities discussed in the literature include shocks in jets $([14,6,15])$, changes in the direction of forward beaming [due, e.g., to helical trajectories of plasma elements ([9]), to a precessing binary black-hole system $([8,21])$, or to a rotation of a helical jet $([24,25])]$, introducing flares due to the lighthouse effect and variability due to colliding relativistic plasma shells $([22,12])$. Thus, variability furnishes important clues into size, structure, physics and dynamics of the radiating source region.

\section{Current Status of the Monitoring}

Since December 2004 we perform a monthly multi-frequency radio monitoring of a sample of blazars at the antennas in Medicina and Noto. For most of the time the lower frequencies of 5, 8, and $22 \mathrm{GHz}$ are observed at Medicina and observations at $43 \mathrm{GHz}$ are performed at Noto.

The sample consists of 38 blazars that were selected to be bright in optical ( $<19$ mag, enough to be monitored by smaller telescopes) and radio wavelength $\left(\mathrm{S}_{1.4}>1 \mathrm{Jy}\right)$ and that were detected in the $\gamma$-rays by EGRET ([13]). Contemporaneous optical and near-IR measurements are organized for many epochs ([7]) by the GLAST-AGILE Support Program (GASP) of the Whole Earth Blazar Telescope $\left(\mathrm{WEBT}^{1}\right)$.

During the last 10 years nearly all sources in our sample display some amount of variability (see Fig. 1). In many cases the flares appear first at the higher frequencies, suggesting that the variability is caused by a shock propagating along the jet, but also simultaneous variations at various frequencies are visible which could be related to changes of the jet viewing angle that lead to variations of the Doppler beaming factor.

Many of the sources have been detected by the ASI $\gamma$-ray satellite AGILE and the NASA Fermi $\gamma$-ray satellite which initiated intensive broadband campaigns. Medicina and Noto data have contributed to more than 25 refereed publications (e.g. [1, 2, 3, 4, 5, 28, 29, 30, 16, 17, 18, 19, 20 , $10,11,26,27]$. Since 2008 the monitoring is one of the IRA-INAF key science programs.

Although the light curves are sometimes interrupted by receiver changes or antenna maintenance we are now in the position to conduct meaningful statistical analysis of the light curves and

\footnotetext{
${ }^{1}$ http://www.oato.inaf.it/blazars/webt/
} 

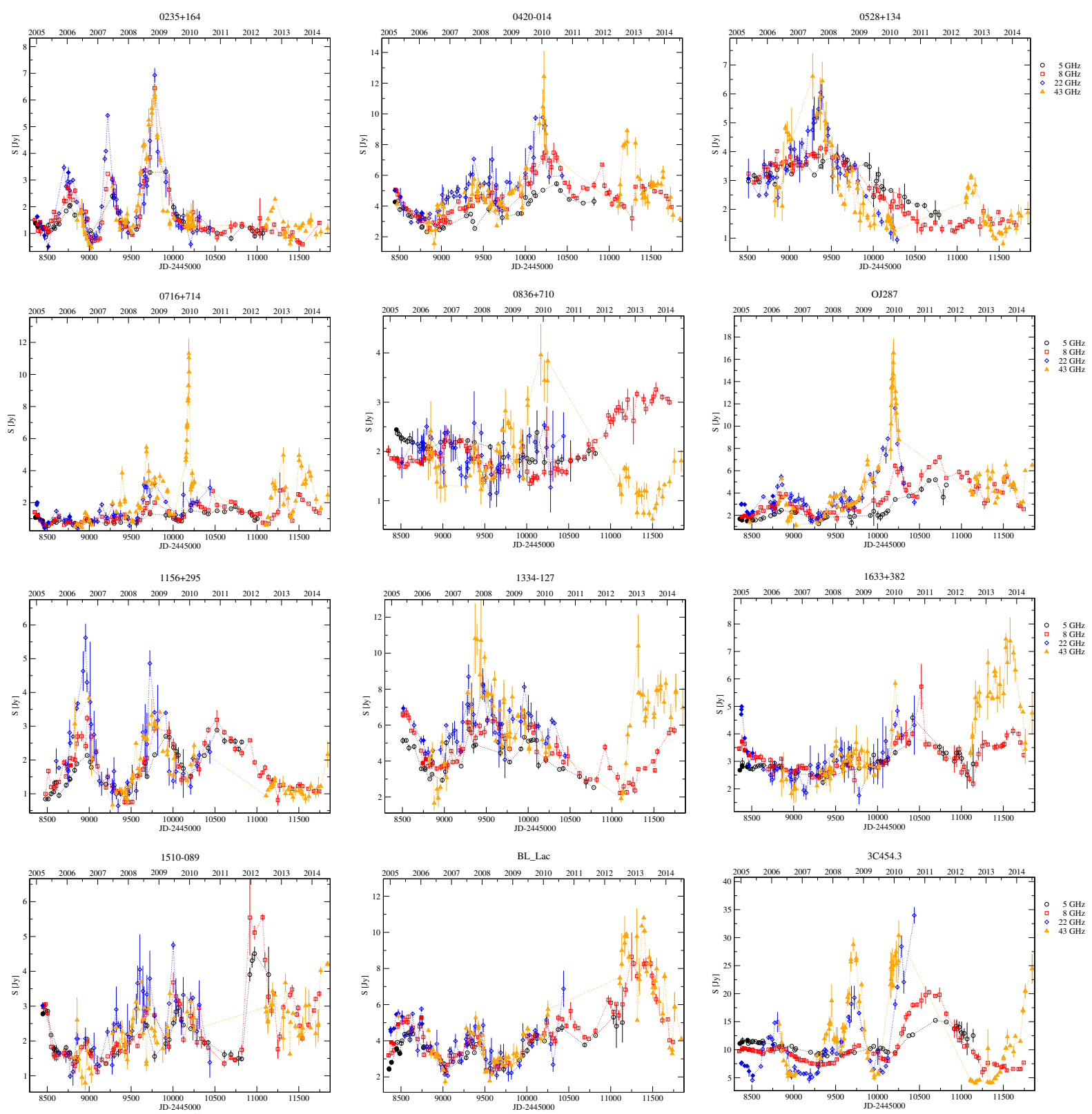

Figure 1: Examples of radio light curves at 5, 8, 22 and $43 \mathrm{GHz}$ of several blazars between December 2004 and Jul 2014 taken with the antennas in Medicina (open symbols) and Noto (filled symbols).

spectra. The monitoring will be continued at least for the life time of the current $\gamma$-ray satellites. All light curves and current flux densities are presented on our web page ${ }^{2}$.

Acknowledgements This work is based on observations with the Medicina and Noto telescopes operated by INAF - Istituto di Radioastronomia

\footnotetext{
${ }^{2}$ http://www3.mpifr-bonn.mpg.de/staff/ubach/bmonit
} 


\section{References}

[1] Abdo, A. A., Ackermann, M., Ajello,M. et al., 2010, Nature, 463, 919

[2] Abdo, A. A., Ackermann, M., Ajello,M., et al. 2010, Science, 329, 817

[3] Abdo, A. A., Ackermann, M., Agudo, I., et al. 2010, ApJ, 716, 30

[4] Abdo, A. A., Ackermann, M., Ajello,M., et al. 2010, ApJ, 721, 1425

[5] Abdo, A. A., Ackermann, M., Ajello,M. et al. 2011, ApJ, 726, 43

[6] Aller, H. D., Aller, M. F., \& Hughes, P. A. 1985, ApJ, 298, 296

[7] Bach, U., Raiteri, C. M., Villata, M., et al. 2007, A\&A, 464, 175

[8] Begelman, M. C., Blandford, R. D., \& Rees, M. J. 1980, Nature, 287, 307

[9] Camenzind, M. \& Krockenberger, M. 1992, A\&A, 255, 59

[10] D’Ammando, F., Pucella, G., Raiteri, C. M., et al. 2009, A\&A, 508, 181

[11] D’Ammando, F., Raiteri, C. M., Villata, M., et al. 2011, A\&A, 529, A145

[12] Guetta, D., Ghisellini, G., Lazzati, D., \& Celotti, A. 2004, A\&A, 421, 877

[13] Hartman, R. C., Bertsch, D. L., Bloom, S. D., et al. 1999, ApJs, 123, 79

[14] Marscher, A. P. \& Gear, W. K. 1985, ApJ, 298, 114

[15] Marscher, A. P. 1996, in ASP Conf. Ser. 100: Energy Transport in Radio Galaxies and Quasars, 45

[16] Raiteri, C. M., Villata, M., Chen, W. P., et al. 2008, A\&A, 485, L17

[17] Raiteri, C. M., Villata, M., Capetti, A., et al. 2009, A\&A, 507, 769

[18] Raiteri, C. M., Villata, M., Aller, M. F., et al. 2011, A\&A, 534, A87

[19] Raiteri, C. M., Villata, M., Smith, P. S., et al. 2012, A\&A, 545, A48

[20] Raiteri, C. M., Villata, M., D’Ammando, F., et al. 2013, MNRAS, 436, 1530

[21] Sillanpää, A., Haarala, S., Valtonen, M. J., Sundelius, B., \& Byrd, G. G. 1988, ApJ, 325, 628

[22] Spada, M., Ghisellini, G., Lazzati, D., \& Celotti, A. 2001, MNRAS, 325, 1559

[23] Urry, C. M. 1999, Astroparticle Physics, 11, 159

[24] Villata, M., Raiteri, C. M., Sillanpaa, A., \& Takalo, L. O. 1998, MNRAS, 293, L13

[25] Villata, M. \& Raiteri, C. M. 1999, A\&A, 347, 30

[26] Vercellone, S., D’ Ammando, F., Vittorini, V., et al. 2010, ApJ, 712, 405

[27] Vercellone, S., Striani, E., Vittorini, V., et al. 2011, ApJ1, 736, L38

[28] Villata, M., Raiteri, C. M., Larionov, V. M., et al. 2008, A\&A, 481, L79

[29] Villata, M., Raiteri, C. M., Gurwell, M. A., et al. 2009a, A\&A, 504, L9

[30] Villata, M., Raiteri, C. M., Gurwell, M. A., et al. 2009b, A\&A, 501, 455 